\title{
A novel scoring system for gastric cancer risk assessment based on the expression of three CLIP4 DNA methylation-associated genes
}

\author{
CHENGGONG HU, YONGFANG ZHOU, CHANG LIU and YAN KANG \\ Department of Critical Care Medicine, West China Hospital of Sichuan University, Chengdu, Sichuan 610041, P.R. China
}

Received January 19, 2018; Accepted April 26, 2018

DOI: $10.3892 / \mathrm{ijo} .2018 .4433$

\begin{abstract}
Gastric cancer (GC) is the fifth most common cancer and the third leading cause of cancer-associated mortality worldwide. In the current study, comprehensive bioinformatic analyses were performed to develop a novel scoring system for GC risk assessment based on CAP-Gly domain containing linker protein family member 4 (CLIP4) DNA methylation status. Two GC datasets with methylation sequencing information and mRNA expression profiling were downloaded from the The Cancer Genome Atlas and Gene Expression Omnibus databases. Differentially expressed genes (DEGs) between the CLIP4 hypermethylation and CLIP4 hypomethylation groups were screened using the limma package in R 3.3.1, and survival analysis of these DEGs was performed using the survival package. A risk scoring system was established via regression factor-weighted gene expression based on linear combination to screen the most important genes associated with CLIP4 methylation and prognosis. Genes associated with high/low-risk value were selected using the limma package. Functional enrichment analysis of the top 500 DEGs that positively and negatively associated with risk values was performed using DAVID 6.8 online and the gene set enrichment analysis (GSEA) software. In total, 35 genes were identified to be that significantly associated with prognosis and
\end{abstract}

Correspondence to: Dr Yan Kang, Department of Critical Care Medicine, West China Hospital of Sichuan University, 37 Guo Xue Xiang, Chengdu, Sichuan 610041, P.R. China

E-mail: huchenggong2017@163.com

Abbreviations: GC, gastric cancer; DEGs, differentially expressed genes; CLIP4, CAP-Gly domain containing linker protein family member 4; TCGA, The Cancer Genome Atlas; EGFR, epidermal growth factor receptor; GEO, Gene Expression Omnibus; GO, gene ontology; GSEA, gene set enrichment analysis; BH, Benjamini-Hochberg; OS, overall survival; KM, Kaplan-Meier; CLDN11, claudin-11, APOD, apolipoprotein D; CHRDL1, chordin-like 1; BMP, bone morphogenetic protein; BMPR, bone morphogenetic protein receptor

Key words: gastric cancer, CAP-Gly domain containing linker protein family member 4 DNA methylation, gene expression, scoring system, functional enrichment analysis
CLIP4 DNA methylation, and three prognostic signature genes, claudin-11 (CLDN11), apolipoprotein D (APOD), and chordin like $1(C H R D L 1)$, were used to establish a risk assessment system. The prognostic scoring system exhibited efficiency in classifying patients with different prognoses, where the lowrisk groups had significantly longer overall survival times than those in the high-risk groups. CLDN11, APOD and CHRDL1 exhibited reduced expression in the hypermethylation and lowrisk groups compare with the hypomethylation and high-risk groups, respectively. Multivariate Cox analysis indicated that risk value could be used as an independent prognostic factor. In functional analysis, six functional gene ontology terms and five GSEA pathways were associated with CLDN11, APOD and $C H R D L 1$. The results established the credibility of the scoring system in this study. Additionally, these three genes, which were significantly associated with CLIP4 DNA methylation and GC risk assessment, were identified as potential prognostic biomarkers.

\section{Introduction}

Gastric cancer (GC) is the fifth most common cancer in both sexes and the third most common cause of cancer-associated mortality worldwide (1). Due to the advances in diagnostic and therapeutic approaches, long-term survival for patients with early stage of GC has improved; however, the outlook for individuals with advanced GC is still disappointing because of poor prognosis and limited treatment options $(2,3)$. Poor prognosis is frequently explained by the lack of early diagnostic biomarkers and effective therapeutic treatments (3). As the prognosis of GC is closely associated with the stage of the disease at diagnosis, novel diagnostic modalities for early stages and novel therapeutics are urgently required (4). Several diagnostic biomarkers, such as aberrantly methylated DNA, have aided the diagnoses and disease monitoring efforts in GC.

Changes in DNA methylation have crucial roles during the early stages of GC; therefore, aberrant DNA methylation is highlighted as the main change differentiating GC subtypes from the very first stage $(2,5,6)$. Over 550 studies have demonstrated that aberrant epigenetic changes of $>100$ genes have crucial roles during the early stages of GC $(7,8)$. For example, a recent report associated promoter methylation of CAP-Gly domain containing linker protein family member 4 (CLIP4) with the increase in severity of gastritis with no metaplasia to 
gastritis with metaplasia and GC, which may be a potentially useful molecular tool for GC risk stratification in endoscopic biopsies (9).

CLIP4, also known as UBASH3A or TULA, is a member of the T-cell ubiquitin ligand family. These proteins can suppress T-cell signaling, facilitate growth factor withdrawal-induced apoptosis in T-cells and promote the accumulation of various activated target receptors, such as T-cell receptors and epidermal growth factor receptors (EGFRs), which can induce cell invasiveness and metastasis (10-12). Additionally, CLIP4 is involved in regulating the expression of several tumor-associated genes, such as spleen associated tyrosine kinase, a member of the protein tyrosine kinase family associated with cell motility and increased cell migration (13-15), and $\mathrm{Cbl}$ proto-oncogene, which downregulates EGFR and activates the epithelial-mesenchymal transition-associated EGFR signaling pathway $(10,16)$. CLIP4 expression is also suggested to stimulate tumor metastasis in certain tumor types (17).

Considering the involvement of CLIP4 in tumor metastasis and the association between its promoter methylation and GC, it is worth exploring the CLIP4 DNA methylation-associated genes that may facilitate further understanding of the function of CLIP4 in the pathogenesis of GC and provide potential diagnostic biomarkers for clinical treatment.

In the present study, large quantities of methylation sequencing information and the mRNA expression profiling data from GC samples published in The Cancer Genome Atlas (TCGA) database were used to screen out the significant differentially expressed genes (DEGs) associated with CLIP4 DNA methylation. A prognostic scoring system was established based on the screened genes and simultaneously validated by the datasets from the independent Gene Expression Omnibus (GEO) database. The reliability of the prognostic scoring system was further validated by correlation analysis between clinical characteristics and prognosis. Functional enrichment analysis of DEGs related to prognosis was performed using gene ontology (GO) and gene set enrichment analysis (GSEA).

\section{Materials and methods}

Samples and datasets. Information of the samples in the training dataset. The training dataset, containing 393 GC samples with both methylation sequencing information (Illumina Human Methylation 450 platform) and mRNA expression profiling data (Illumina platform), was downloaded from the TCGA database (https://portal.gdc.cancer.gov/) on February 10, 2017. The 393 patients with GC consisted of 258 males and 135 females with $65.761 \pm 10.706$ years [mean \pm standard deviation (SD)]. There were 251 Caucasian patients, 107 non-Caucasian patients, and the race of the remaining patients was unavailable. There were 52 stage I, 125 stage II, 173 stage III, 32 stage IV cases and the remaining cases were at unknown stages. A total of 152 patients had succumbed to disease when data was submitted, with an average survival time of $438.88 \pm 384.35$ days. Information of the samples in the training dataset is summarized in Table I.

Information of the samples in the validation dataset. The validation dataset, containing 157 GC samples with
Table I. Information of samples in training and validation datasets.

\begin{tabular}{lcc}
\hline Parameter & $\begin{array}{c}\text { TCGA } \\
(\mathrm{n}=393)\end{array}$ & $\begin{array}{c}\text { GSE30601 and } \\
\text { GSE15460 } \\
(\mathrm{n}=157)\end{array}$ \\
\hline Age (mean \pm SD) & $65.761 \pm 10.706$ & $63.242 \pm 12.607$ \\
Sex (male/female) & $258 / 135$ & $100 / 57$ \\
Stage $(\mathrm{I} / \mathrm{II} / \mathrm{III} / \mathrm{IV})$ & $52 / 125 / 173 / 32$ & $23 / 25 / 59 / 20$ \\
$\begin{array}{l}\text { Overall survival } \\
\text { (mean } \pm \text { SD) }\end{array}$ & $438.88 \pm 384.35$ & $699.88 \pm 728.17$ \\
Radiotherapy (yes/no) & $77 / 366$ & \\
$\begin{array}{l}\text { Chemotherapy (yes/no) } \\
\text { Targeted molecular therapy } \\
\text { (yes/no) }\end{array}$ & $183 / 260$ & \\
\hline
\end{tabular}

TCGA, The Cancer Genome Atlas; SD, standard deviation.

methylation sequencing information (GSE30601; Illumina HumanMethylation27 BeadChip; ncbi.nlm.nih.gov/geo/query/ acc.cgi?acc $=$ GSE30601) and mRNA expression profiling data (GSE15460; Affymetrix GeneChip Human Genome U133 Plus $2.0 ;$;ncbi.nlm.nih.gov/geo/query/acc.cgi?acc=GSE15460), was downloaded from the GEO database (18). The 157 GC patients consisted of 100 males and 57 females with $63.242 \pm 12.607$ years (mean \pm SD). There were 23 stage I, 25 stage II, 59 stage III and 20 stage IV cases, and the remaining stages were unavailable. The average survival time for 81 patients was $699.88 \pm 728.17$ days (mean $\pm \mathrm{SD}$ ). Information on the samples in the validation dataset is summarized in Table I.

Selection of candidate expression factors. Samples were divided into CLIP4 hypermethylation and CLIP4 hypomethylation groups according to the median CLIP4 methylated value of 0.326 . The gene expression differences between the two groups were compared using the limma package in the R software 3.3.1 (19), and genes with $\mid \log$ fold change (FC) $\mid>1$ and Benjamini-Hochberg (BH)-adjusted $\mathrm{P}<0.01$ were considered to be significant DEGs. Subsequently, survival analysis associated with these DEGs was performed by Kaplan-Meier analysis and univariate Cox regression analysis using the 'survidiff' function in the survival package of $R$ 3.3.1 $(20,21)$ and $P<0.05$ was set as the significance threshold. As mortality of patients with an overall survival (OS) of $<30$ days may due to other factors, these patients and those without survival data were excluded from the survival analysis. The KM diagram was generated using 'ggsurvplot'. The top three genes that were significantly associated with OS $(\mathrm{P}<0.005)$ were selected as signature genes.

Establishment of the risk assessment model. Each risk value was calculated as a linear combination of the mRNA expression value (expr) following weighting by regression coefficients $(\beta)$ (22-24). The risk score for each patient was calculated according to the following formula:

Risk score $=\beta_{\text {genel } 1} \times \operatorname{expr}_{\text {gene1 }}+\beta_{\text {gene2 } 2} \mathrm{x}$ expr $r_{\text {gene2 } 2}+\beta_{\text {gene3 }} \mathrm{x}$ expr $r_{\text {gene } 3}$ $\beta$ represents the gene risk coefficient, expr represents the gene expression level and gene1, gene2 and gene 3 represents the 


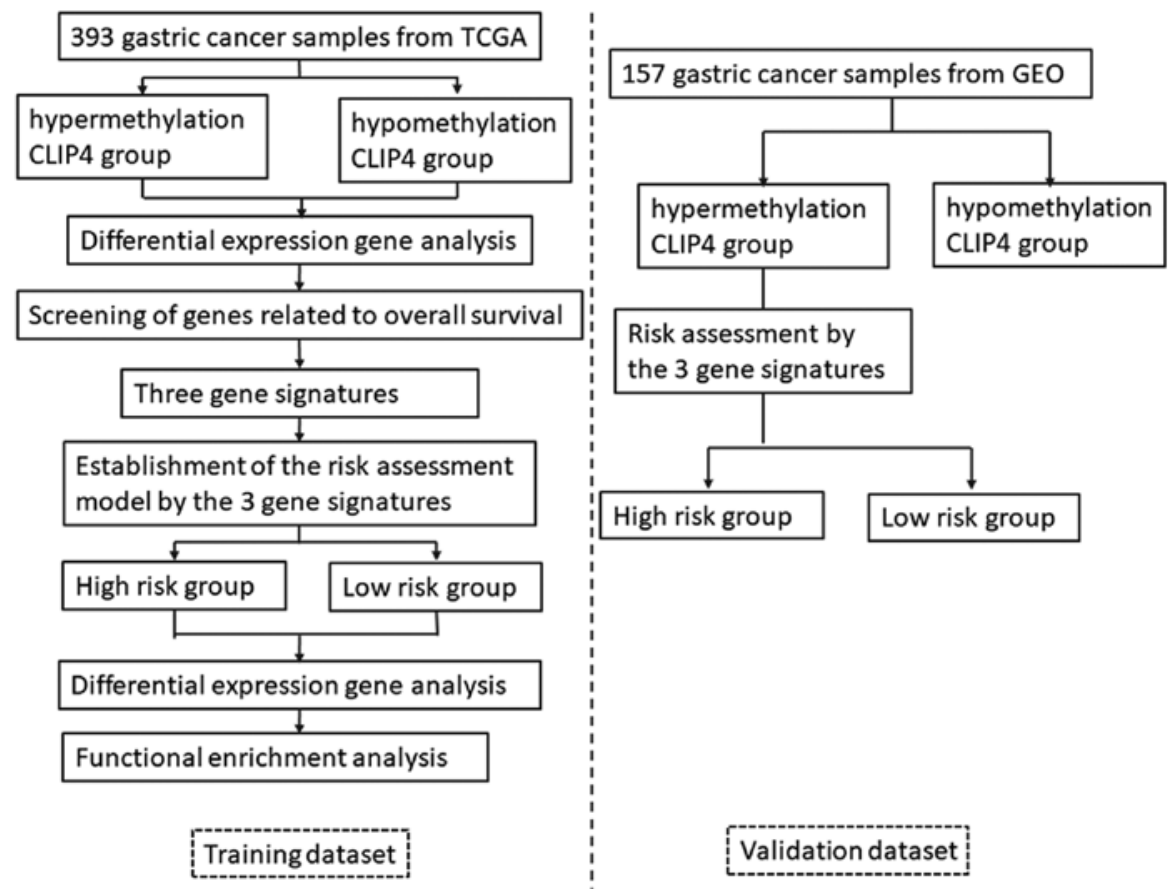

Figure 1. Workflow of the present study. TCGA, The Cancer Genome Atlas; GEO, Gene Expression Omnibus; CLIP4, CAP-Gly domain containing linker protein family member 4 .

three genes. The high- and low-risk groups were classified based on the median of the risk values.

Functional annotation of the prognostic genes. BH-adjusted $\mathrm{P}<0.01$ was used as the threshold to screen out genes significantly associated with high- and low-risk groups using the limma package. According to the association between the genes and their risk values, the genes positively or negatively associated with the risk value were defined as the high-risk group and high expression genes, or low-risk group and low expression genes, respectively. The top 100 (alternatively 50) genes with high and low expression were chosen to generate a heatmap plot using the ggplot 2 drawing package. Subsequently, functional enrichment analysis and mapping of the top 500 genes with high and low expression levels were performed using the DAVID 6.8 online software (https://david.ncifcrf. gov) $(25,26)$.

Verification of gene functions was performed via the screening standard, nominal $\mathrm{P}<0.05$, using the GSEA software (software.broadinstitute.org/gsea/index.jsp) (27,28). GSEA analysis is a statistical method for calculating the enrichment of a gene list in a pathway. Briefly, all the genes in a particular gene list are scored and ranked by a statistical method based on their expression levels. The primary result of GSEA is the enrichment score (ES), which reflects the degree to which a pathway is overrepresented at the top or bottom of the ranked list of genes. The ES was calculated by walking down the ranked list of genes, increasing a runningsum statistic when a gene is in the pathway while decreasing it when it is not. The ES is the maximum deviation from zero encountered in walking the list. The score at the peak of the plot is the ES for the gene set. Gene sets with a distinct peak at the beginning or end of the ranked list are generally the most interesting. For this process, the significant P-values calculated by permutation 1,000 times determined whether the genes were enriched or not.

Statistical analysis. In addition to the statistical methods noted above, the statistical method used in this study was t-test. Univariate Cox regression was used to analyzed the clinical features and risk score for association with patient OS. Multivariate Cox regression analysis were conducted to evaluate whether the clinical features and risk score was independent of other clinical variables, with hazard ratios were calculated. $\mathrm{P}<0.05$ was considered to indicate a statistically significant difference using R 3.3.1.

\section{Results}

Identification and validation of a three-gene prognostic signature in two datasets. The workflow of the current study is presented in Fig. 1. In the training dataset, the samples were divided into the hypermethylated and hypomethylated groups, each with 168 individuals, based on 0.326 as the median of the CLIP4 methylated values. The gene expression differences between the two groups were compared using the limma package, and 279 DEGs were filtered via the threshold $\mid \operatorname{logFCl}>1$ and $\mathrm{BH}$-adjusted $\mathrm{P}<0.01$. This revealed the expression level of 35 genes were significantly associated with prognosis, obtained using the univariate Cox regression analysis (data not shown). High expression levels of 32 genes out of 35 were associated with shorter OS; while higher expression levels of the other 3 genes were associated with longer OS. The top three genes with lowest $\mathrm{P}$-value $(\mathrm{P}<0.005)$, claudin-11 (CLDN11), apolipoprotein D (APOD), and chordin like $1(C H R D L 1)$, were selected as the prognostic gene signatures in the CLIP4 DNA hypermethylation patients. 


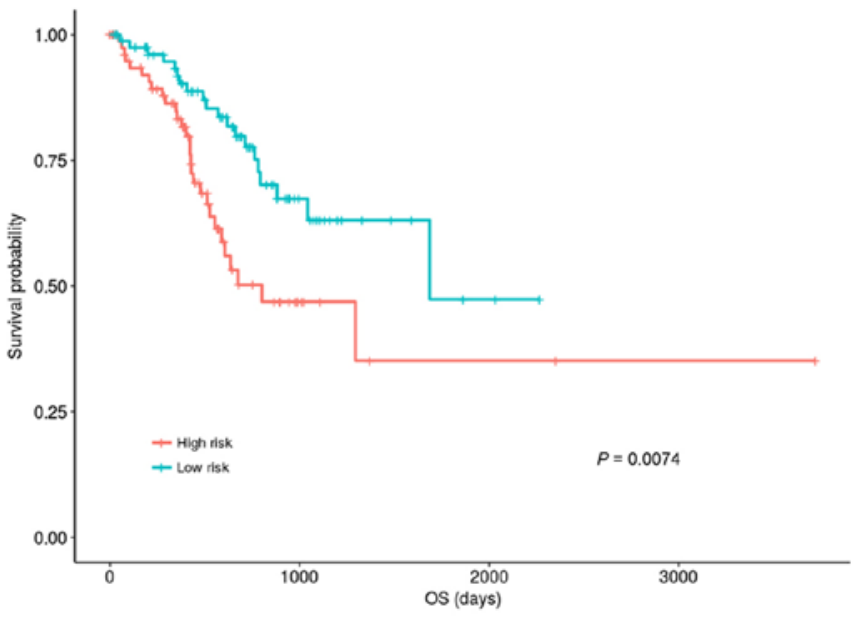

Figure 2. Kaplan-Meier plot of the OS for the low- and high-risk group in the training dataset. Samples were divided into hypermethylation group and hypomethylation according to the median of the CAP-Glydomain containing linker protein family member 4 methylation values. Differentially expressed genes analysis was performed for the two groups. Three genes significantly associated with OS $(\mathrm{P}<0.005)$ were selected as the prognostic gene signatures. A risk assessment system was established based on the expression level of claudin-11, apolipoprotein D and chordin like 1. The patients in hypermethylation group were divided into high- and low-risk groups according to median risk score. The prognosis of the patients in the low-risk group was significantly better than those in the high-risk group. OS, overall survival.

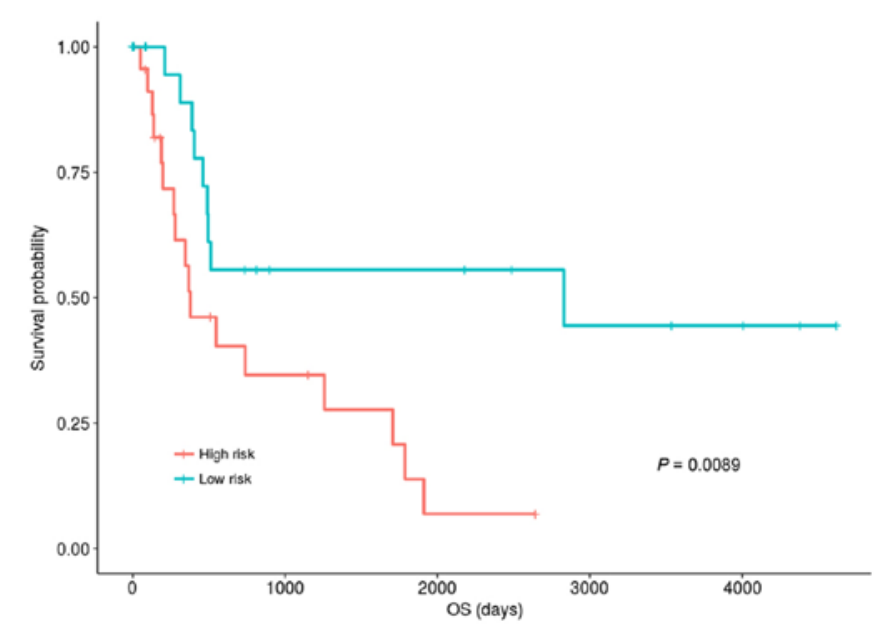

Figure 3. Kaplan-Meier plot of OS for the low- and high-risk group in the validation dataset. Samples were divided into hypermethylation group and hypomethylation according to the median of the CAP-Gly domain containing linker protein family member 4 methylation values. A risk assessment system was established and patients in hypermethylation group were divided into high- and low-risk groups according to median risk score. The prognosis of the patients in the low-risk group was significantly better than those in the high-risk group. OS, overall survival.

Using these three genes, a risk assessment system for cancer patients was established via regression-weighted gene expression based on linear combination. The risk score for each patient was calculated according to the following formula: Risk score $=0.30 \times C L D N 11$ expression $+0.16 \times A P O D$ expression $+0.14 \times$ CHRDL1 expression.

Patients in the hypermethylation group were divided into high- and low-risk groups according to median risk score of 0.4289. Fig. 2 indicated that the OS of the patients in the low-risk group was significantly improved compared with
Table II. Univariate and multivariate Cox analysis of clinical data with overall survival of samples in the training dataset.

\begin{tabular}{|c|c|c|c|c|}
\hline \multirow[b]{2}{*}{ Variable } & \multicolumn{2}{|c|}{ Univariate Cox } & \multicolumn{2}{|c|}{ Multivariate Cox } \\
\hline & P-value & HR & P-value & HR \\
\hline Age $(>60 / \leq 60)$ & 0.0745 & 1.0142 & & \\
\hline Sex (male/female) & 0.0899 & 1.3700 & & \\
\hline Race (white/non-white) & 0.6412 & 1.0352 & & \\
\hline Chemotherapy (yes/no) & 0.0646 & 0.7302 & & \\
\hline $\begin{array}{l}\text { Targeted molecular } \\
\text { therapy (yes/no) }\end{array}$ & 0.0300 & 0.6886 & 0.7461 & 0.9342 \\
\hline Radiotherapy (yes/no) & 0.0013 & 0.4544 & 0.0296 & 0.5260 \\
\hline $\begin{array}{l}\text { Risk value } \\
(>\text { median/smedian })\end{array}$ & 0.0089 & 0.4635 & 0.0065 & 0.6091 \\
\hline
\end{tabular}

HR, hazard ratio.

those in the high-risk group ( $\mathrm{P}=0.00744$; $\mathrm{KM}$ analysis and log-rank test). The OS median values of the low- and high-risk groups were 538.5 days and 422 days, respectively.

The median of methylation in the training set was used as the standard for dividing samples in validation dataset into hypomethylation and hypermethylation groups. In the validation dataset, 48 patients were classified into the hypermethylation group. The risk scores of samples in the hypermethylation group of validation set were calculated according to the risk assessment system, and the samples were also divided into high-risk group and low-risk group according to the median risk score. Using the median risk score of 5.04 as the dividing point, the samples were divided into high- and low-risk groups with 24 individuals in each group. Fig. 3 demonstrated that the OS of the patients in the low-risk group was significantly improved compared with those in the highrisk group $(\mathrm{P}=0.0083, \mathrm{KM}$ analysis and log-rank test). The OS median values of the low- and high-risk groups were 462 days and 345 days, respectively. This result suggested that the risk assessment was also effective in the validation dataset.

Clinical and molecular features of the low- and high-risk patients with CLIP4 promoter methylation. Fig. 4 indicated that the expression levels of the three DEGs, CLDN11, $A P O D$ and $C H R D L 1$, in the hypermethylation group were significantly lower than those in the hypomethylation group. The P-values, determined via Student's t-test, were $1.09 \times 10^{-13}$ $(C L D N 11), 4.12 \times 10^{-8}(A P O D)$ and 0.00128 (CHRDL1). Further, their expression levels were significantly different between the high- and low-risk groups in the CLIP4 DNA hypermethylation patients, as presented in Fig. 5 .

The independence of the three important factors was also evaluated. In the training dataset, univariate Cox regression analysis of patient age, sex, race, chemotherapy, targeted molecular therapy, radiotherapy and risk value were analyzed for association with patient OS. Targeted molecular therapy, radiotherapy and risk value were associated with GC patient overall survival time $(\mathrm{P}<0.05$; Table II). Multivariate Cox analysis was also performed on targeted molecular therapy, 

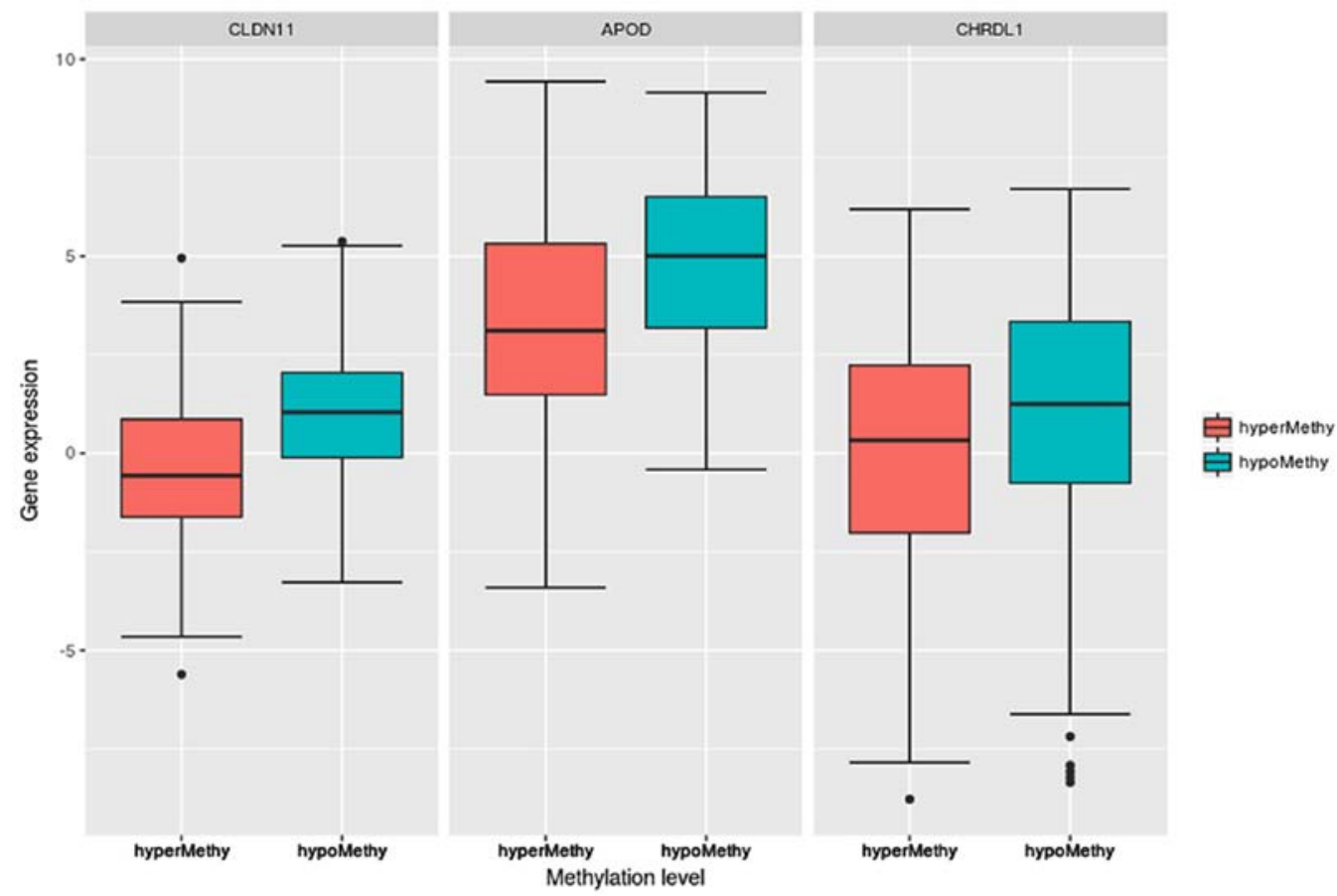

Figure 4. Boxplots of the $C L D N 11, A P O D$ and $C H R D L 1$ expression levels in the hypomethylation and hypermethylation groups. The expression levels of the three genes in the hypermethylation group were significantly lower than those in the hypomethylation group: $C L D N 11\left(\mathrm{P}=1.09 \times 10^{-13}\right), A P O D\left(\mathrm{P}=4.12 \times 10^{-8}\right)$, and $C H R D L 1\left(\mathrm{P}=1.28 \times 10^{-3}\right)$. CLDN11, claudin 11; APOD, apolipoprotein D; CHRDL1, chordin like 1.
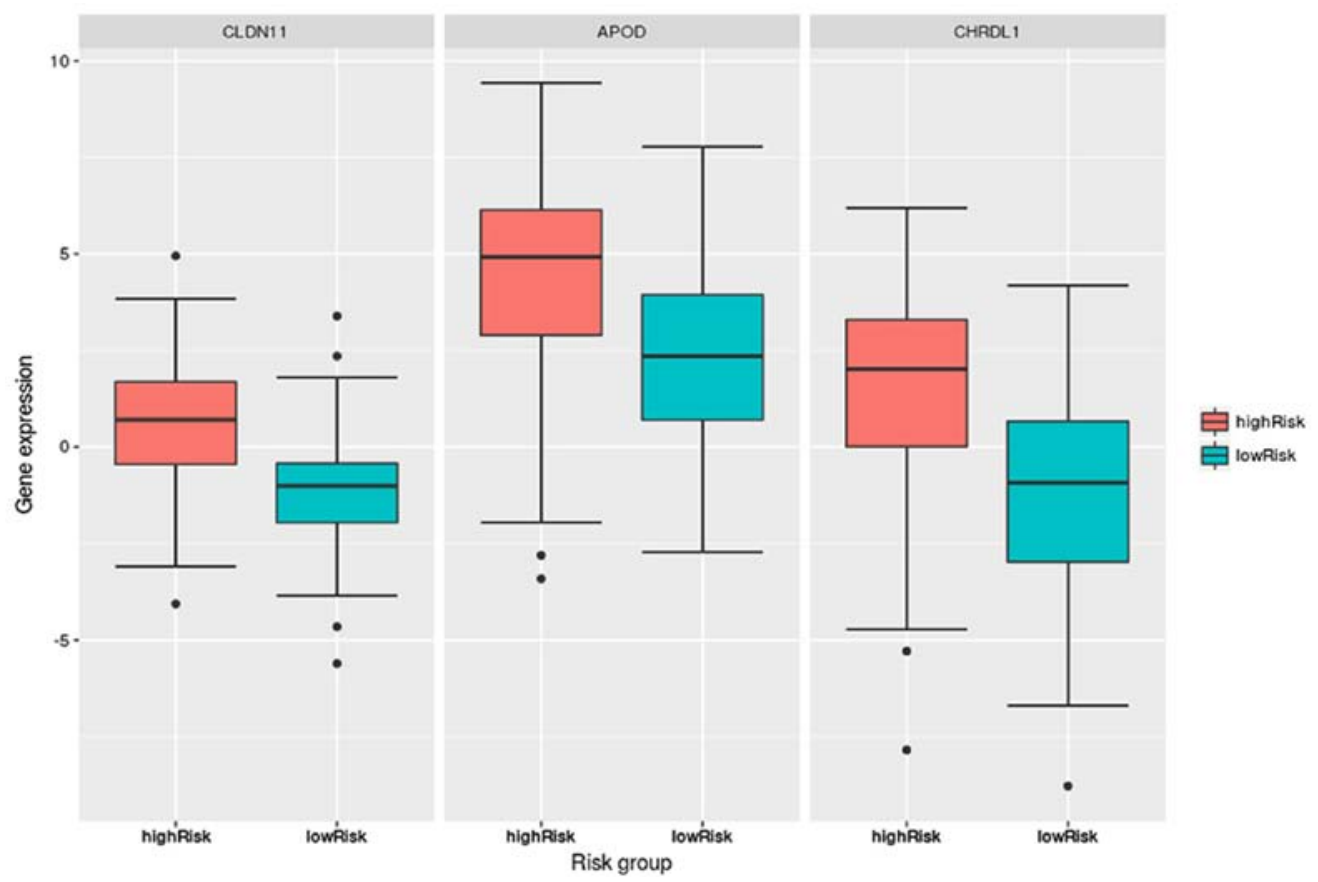

Figure 5. Boxplots of the CLDN11, APOD and CHRDL1 expression levels in the low- and high-risk groups. The expression levels of the three genes were significantly different between the high- and low-risk groups: $C L D N 11\left(\mathrm{P}=1.48 \times 10^{-10}\right), A P O D\left(\mathrm{P}=1.71 \times 10^{-6}\right)$ and $C H R D L 1\left(\mathrm{P}=2.17 \times 10^{-8}\right)$. CLDN11, claudin 11; APOD, apolipoprotein D; CHRDL1, chordin like 1.

radiotherapy and risk value. The results showed radiotherapy and risk value to be independent prognostic factors. Fig. 6 demonstrated the risk values, OS and expression levels of the three genes in the training (left) and validation (right) datasets.

Association of the three-gene signature prognosis values with pathological stage and radiotherapy. CLIP4 is reported to be closely associated with to cancer development $(9,29)$. Therefore, prognostic differences between the high- and lowrisk groups in patients with CLIP4 hypermethylation during different stages were explored in the current study. Fig. 7 results indicated no significant prognostic difference between the high- and low-risk groups during stages 1 and 2, potentially due to an insufficient amount of total statistical samples, even 


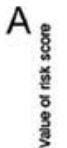

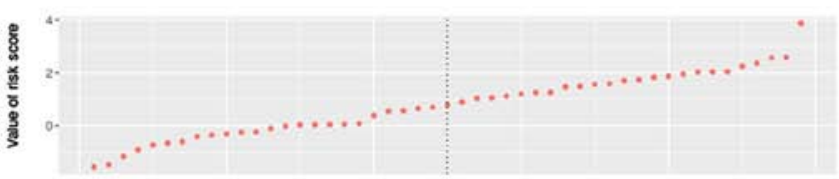

A1

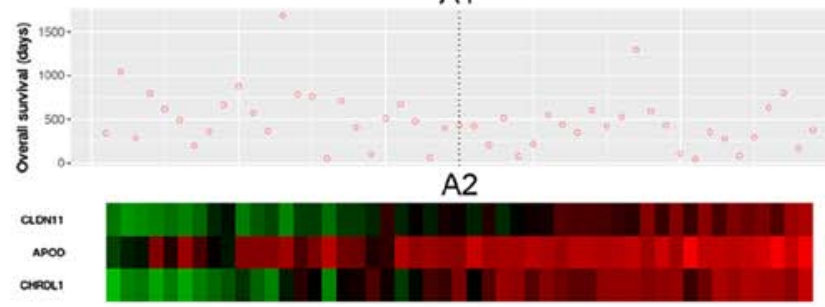

A3
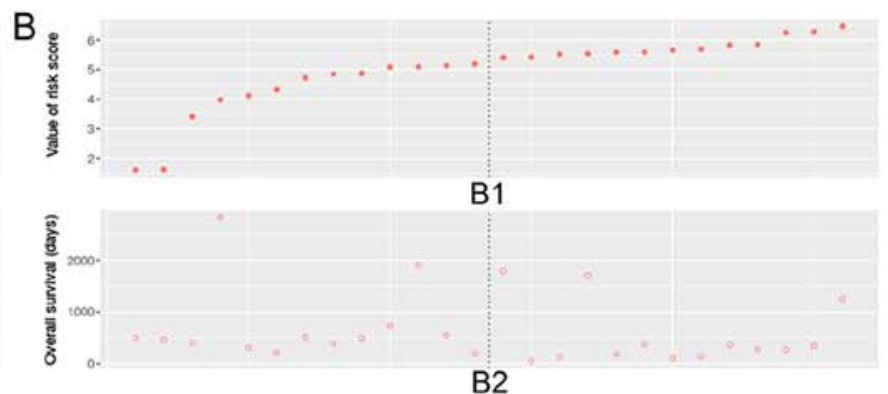

Oow
OROO

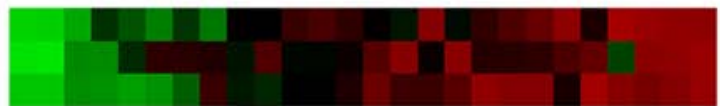

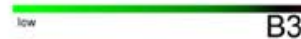

Figure 6. Distribution of risk score, overall survival and gene expression in the (A) training and (B) validation datasets. A1 and B1 indicate the gradually rising trend in patient corresponding risk values in the training and validation datasets, respectively. A2 and B2 show the corresponding overall survival of patients. A3 and B3 indicate the expression levels of these three genes in patients, where the transition from green to red represents the increase in gene expression levels. CLDN11, claudin 11; APOD, apolipoprotein D; CHRDL1, chordin like 1.
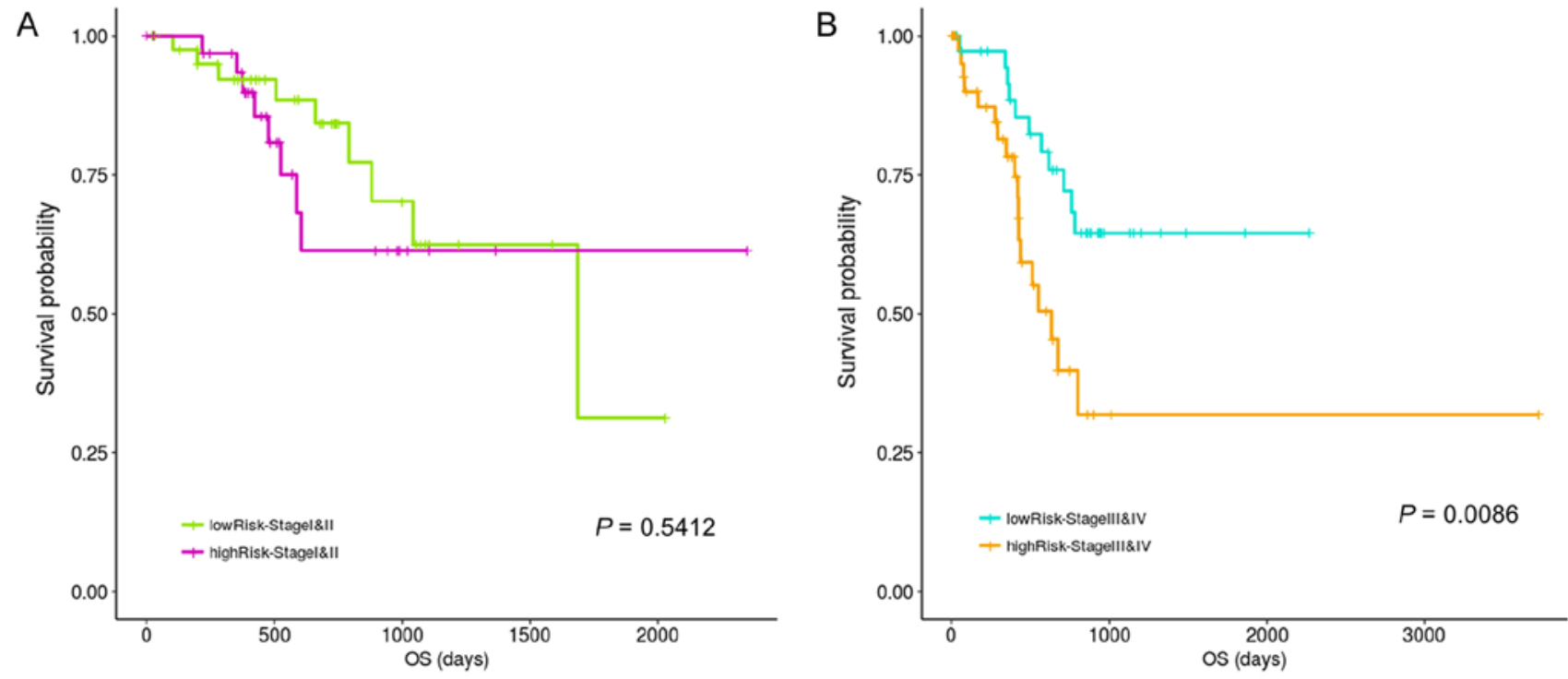

Figure 7. Stratification analysis of the clinical stage and risk score. No significant prognostic difference between the two risk groups in stage 1 and 2 patients However, a significant difference was observed between the high- and low-risk groups at stages 3 and 4 . OS, overall survival.

though a difference in the trend could be observed. However, significant differences were observed between the high- and low-risk groups in stage 3 and 4 patients.

The impact of radiotherapy on risk assessment was also examined. Fig. 8 results indicated significant prognostic differences between the high- and low-risk groups for nonradiotherapy patient, with no difference in survival for those that had received radiotherapy.

Functional enrichment analysis of the DEGs related to prognosis. In the training dataset, DEGs were screened between the high- and low-risk groups using $\mathrm{BH}$-adjusted $\mathrm{P}<0.01$ as the threshold via the limma package. The top 500 DEGs that were positively and negatively associated with risk value were functionally enriched and DEG expression patterns were analyzed using hierarchical clustering. In Fig. 9, the upper and lower heatmaps represented 500 genes that were positively and negatively associated with risk values, respectively. Fig. 9 also presents the top 12 biological process terms involving DEGs that had a significant positive or negative association with the risk values. Functional enrichment analysis showed that CLDN11, APOD and CHRDL1 are involved in six functional terms ('cell adhesion', 'cell-cell adhesion', 'nervous system development', 'signal transduction', 'cell proliferation' and 'negative regulation of cell proliferation').

The pathways significantly enriched in the high- and low-risk groups were stored in the GSEA folder, of which six and four pathways were respectively enriched in the two groups shown in Fig. 10. The increasing curve trends demonstrated that the top-ranked genes were preferentially enriched in the aforementioned pathways. However, the declining curves showed a gradual decrease in the number of genes that were enriched in pathways. CLDN11, APOD and $C H R D L 1$ are involved in a total of five GSEA pathways 

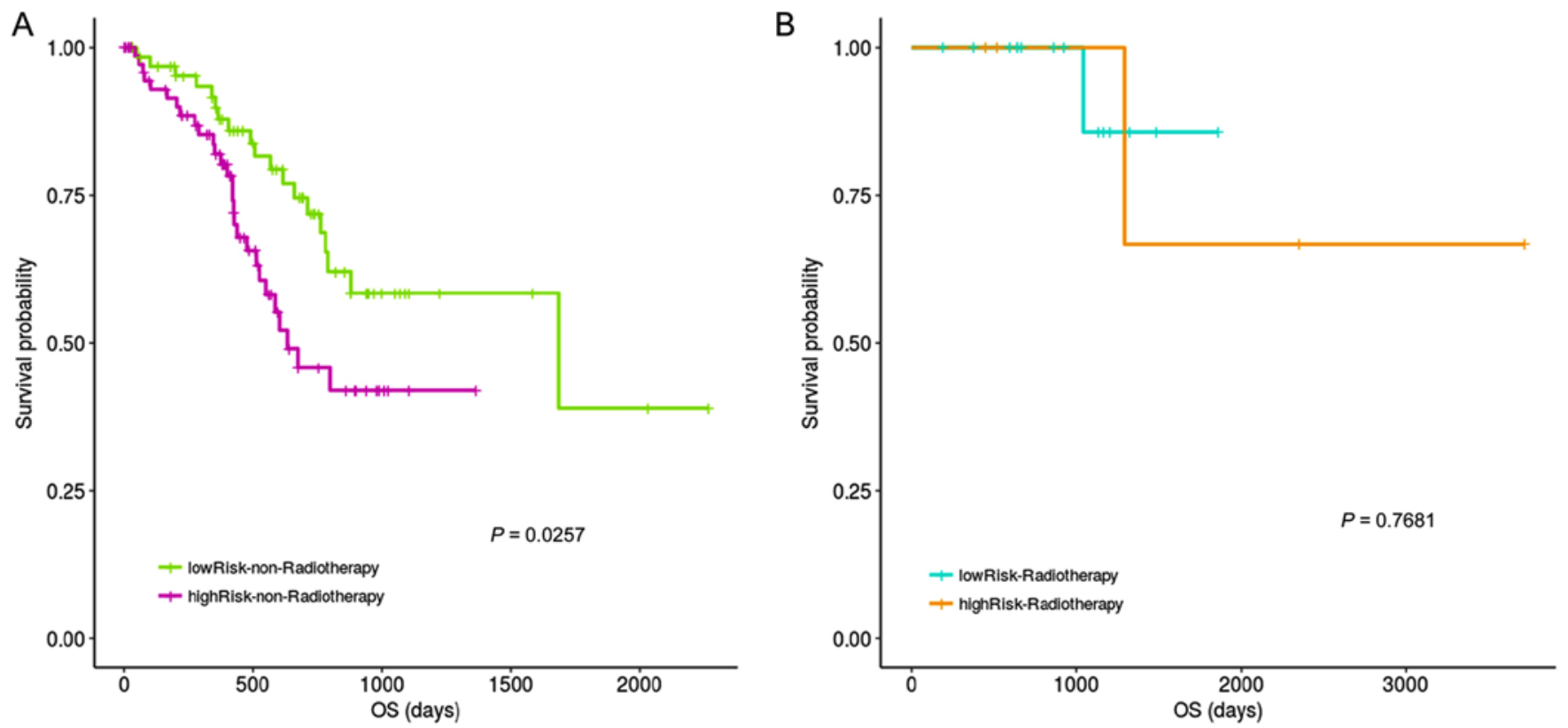

Figure 8. Stratification analysis of radiotherapy and the risk score. (A) Significant prognostic differences were observed between the high- and low-risk groups for non-radiotherapy samples and (B) no significant prognostic difference was observed between high- and low-risk groups for radiotherapy samples. OS, overall survival.
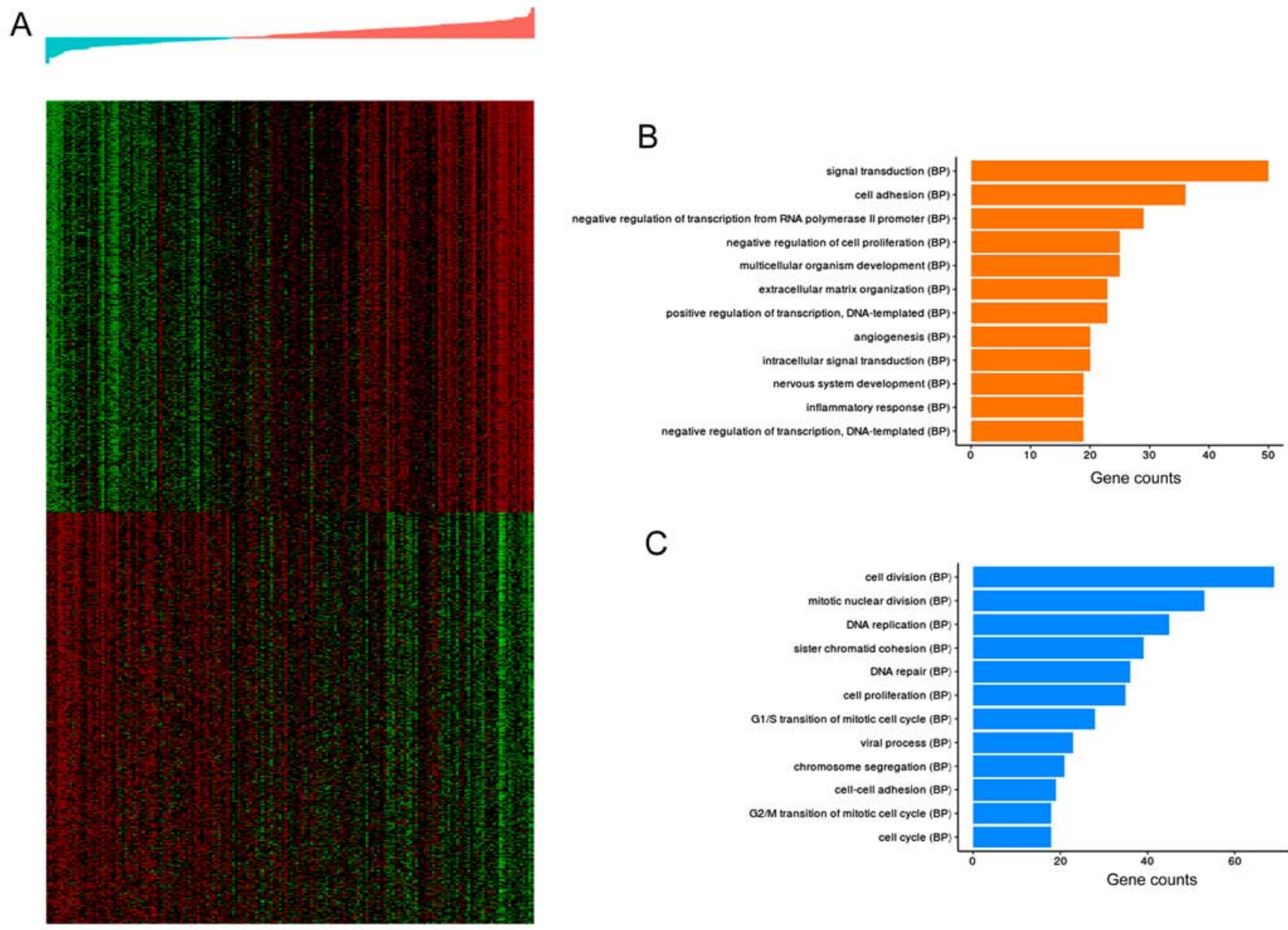

\section{C}

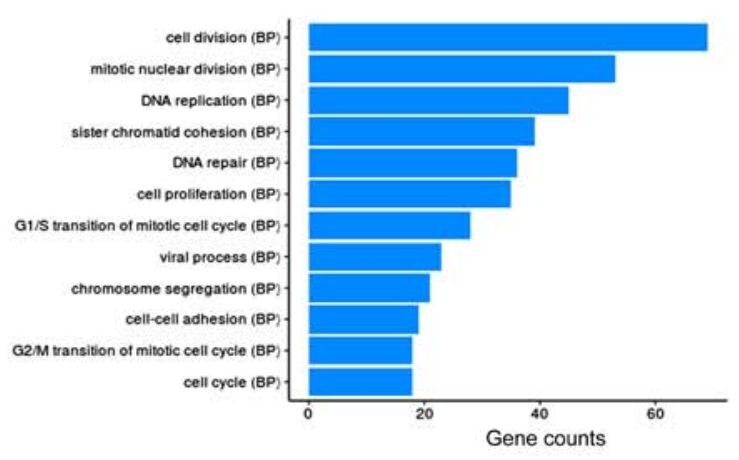

Figure 9. Functional annotation of the DEGs in high- and low-risk samples. (A) Heatmap of the top 500 positively-and negatively-associated genes. Transition from green to red represents the increase in gene expression levels. The top panel represents the distribution of the risk values, increasing from left to right. (B) Barplots of the significantly enriched GO (BP) terms of the top 500 DEGs positively associated with risk. (C) Barplots of the significantly enriched GO (BP) terms of the top 500 DEGs negatively associated with risk. Column length: gene counts. DEG, differentially expressed genes; GO, gene ontology; BP, biological process. 
A Enrichment plot: HALLMARK_APICAL_JUNCTION
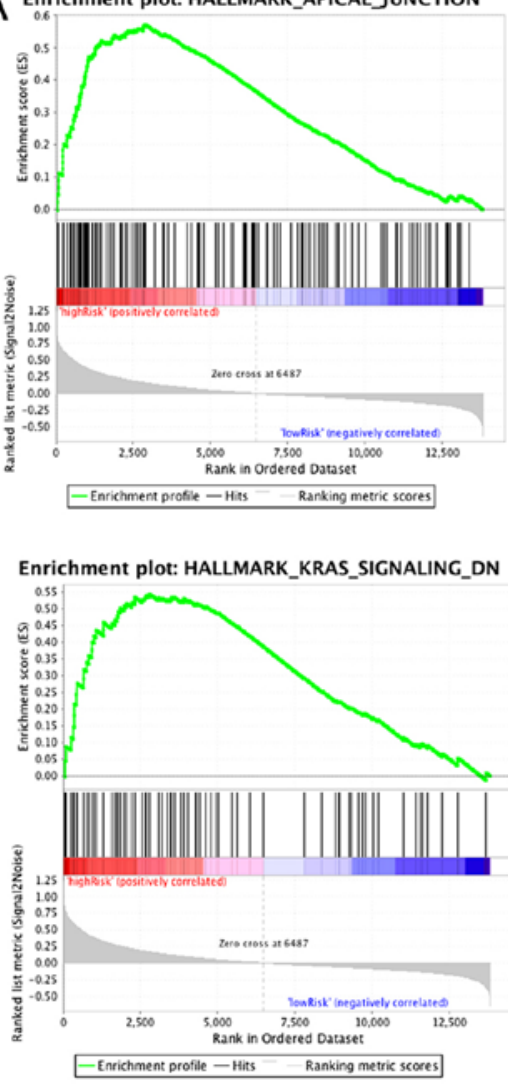

B
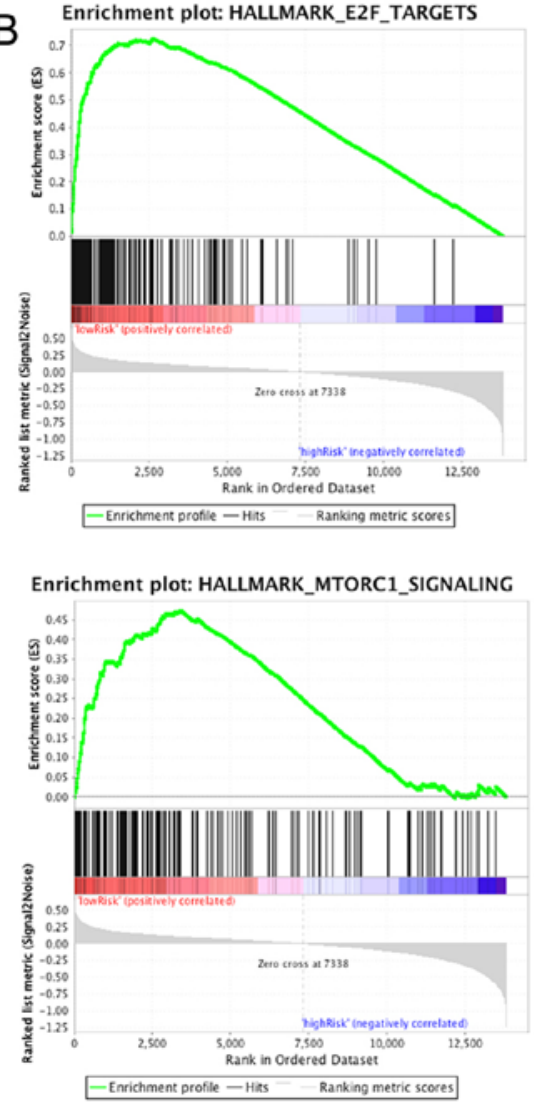
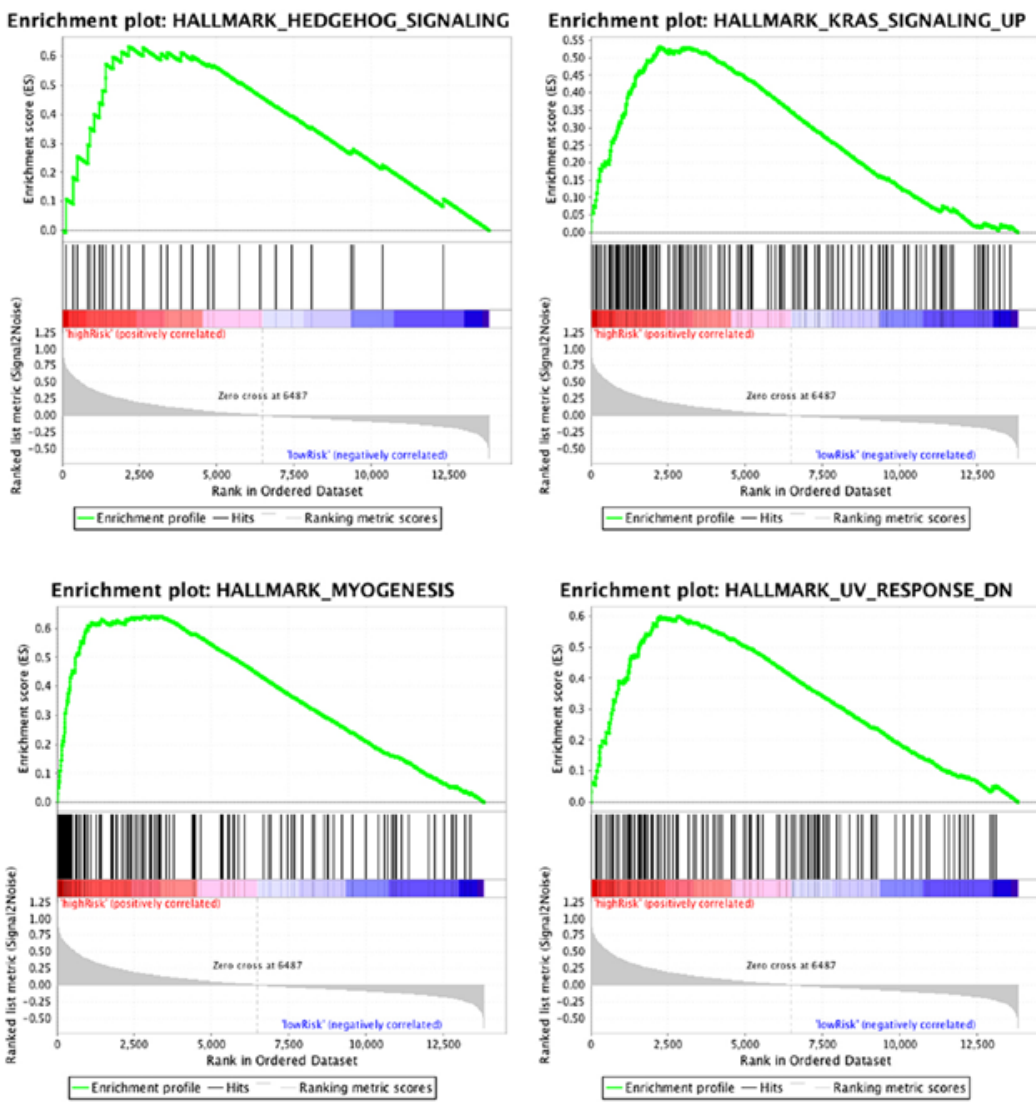

Enrichment plot: HALLMARK_G2M_CHECKPOINT
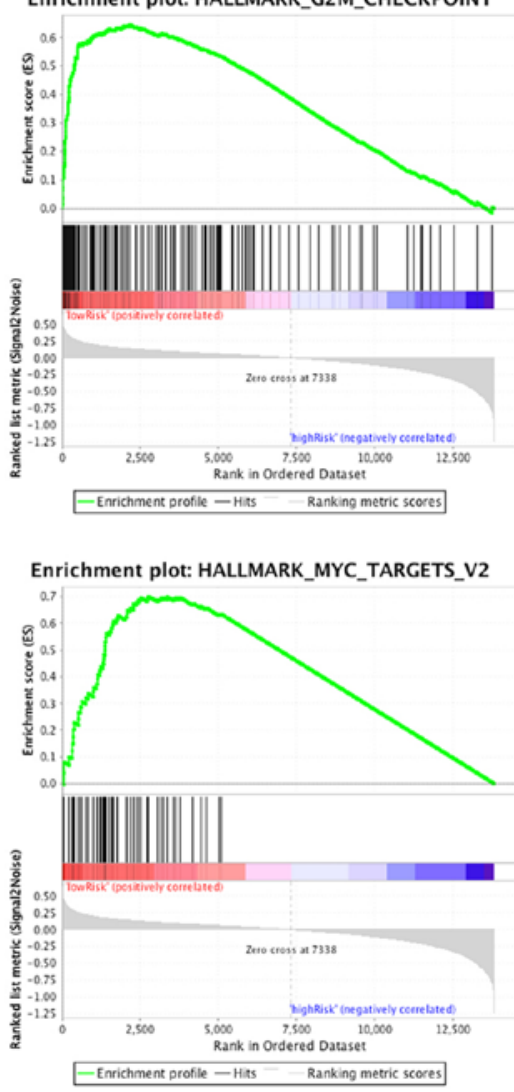

Figure 10. Top 6 enriched pathways $(\mathrm{P}<0.01)$ in $(\mathrm{A})$ the high-risk group, and $(\mathrm{B})$ the top 4 enriched pathways $(\mathrm{P}<0.01)$ in the low-risk group analyzed using gene set enrichment analysis. The top curve in the graph represents the ES, which reflects the degree to which a pathway is overrepresented at the top or bottom of the ranked list of genes. The ES was calculated by walking down the ranked list of genes, increasing a running-sum statistic when a gene is in the pathway while decreasing it when it is not. The ES is the maximum deviation from zero encountered in walking the list. The score at the peak of the plot is the ES for the gene set. Gene sets with a distinct peak at the beginning or end of the ranked list are generally the most interesting. The x-axis represent all the genes in a particular gene list which were firstly scored and ranked by a statistical method based on their expression levels. ES, enrichment score. 
('Hallmark_Apical_Junction', 'Hallmark_Mtorc1_Signaling', 'Hallmark_Kras_Signaling_Up', 'Hallmark_Hedgehog_ Signaling' and 'Hallmark_Kras_Signaling_Dn').

\section{Discussion}

Changes in DNA methylation have crucial roles during the early stages of GC; therefore, aberrant DNA methylation is a major change differentiating GC subtypes from the very first stage $(2,5,6)$. Among the 100 genes with aberrant epigenetic changes that have crucial roles during the early stages of GC (7,8), CLIP4 is of particular interest as it is involved in tumor metastasis and its promoter methylation is associated with an increase in GC severity (9). Thus, it is worth exploring CLIP4 DNA methylation-associated genes that may help to further understand the function of CLIP4 in the pathogenesis of GC and provide potential diagnostic biomarkers for clinical treatment.

In the present study, the methylation sequencing information and mRNA expression profiling data of $393 \mathrm{GC}$ samples were downloaded from the TCGA database and used as the training dataset to screen for significant DEGs associated with CLIP4 DNA methylation. The TCGA database is a large-scale cancer genomics project that has generated an overwhelming amount of cancer genomics data from multiple technical platforms that is of great value to cancer research (30). Consequently, several elegant studies have demonstrated the value of analyzing networks based on this database (31). Of the DEGs with differential expression between samples with CLIP4 hyper- and hypo-methylation, 35 genes were identified to be significantly associated with GC prognosis (OS) using the univariate Cox regression analysis, out of which three genes (CLDN11, APOD and $C H R D L 1)$ were significantly associated with OS. These genes were selected as prognostic gene signatures to establish a risk assessment system, which indicated that the prognosis of patients in the low-risk group was significantly improved compared with those in the high-risk group. Reliability tests were performed using a validation dataset that included the methylation sequencing information and mRNA expression profiling data of the other $157 \mathrm{GC}$ samples (18) downloaded from the GEO database (32).

All three genes were associated with CLIP4 DNA methylation and prognosis of patients with GC. CLDN11 is a member of the claudin family of proteins, which are transmembrane proteins that have crucial roles in tight junction (TJ) formation and function (33). TJs are intercellular junctions that are crucial for paracellular solute transport and cell polarity maintenance. Tumor cells commonly exhibit structural and functional deficiencies in their TJs (34), and aberrant expression of claudin proteins is also observed in various cancer types $(35,36)$. For example, CLDN11 is silenced in GC via hypermethylation of its promoter region, which contributes to GC by increasing cellular motility and invasiveness (33). DEGs associated with risk were confirmed to be enriched in 'cell adhesion' and 'cell-cell adhesion' GO terms, and enriched in the 'Hallmark_Apical_Junction' pathway in GSEA analysis. ApoD is a small, soluble lipid carrier expressed in most human tissues, particularly in the glia of the nervous system $(37,38)$. It is elevated in various pathological situations, particularly in patients with nervous system diseases, including Alzheimer's disease, Parkinson's disease and schizophrenia $(39,40)$. It has also been indicated in the transport of membrane lipids and may be involved in the clearance and/or repair of damaged membranes, potentially by the quenching of harmful materials released by neurons and glial cells in response to damage or recruitment of lipids to expanding membranes (37). GO analysis indicated that the DEGs were enriched in 'nervous system development'. CHRDL1 is a secreted protein that acts as an antagonist of bone morphogenetic protein (BMP), which activates BMP receptor (BMPR) II $(41,42)$. The activation of intracellular signaling via BMPR induces a series of responses, including proliferation, migration and invasion in various tumor types (43). Furthermore, direct experimental evidence suggests that CHRDL1 has an important role in embryonic cell differentiation and in the adult brain $(44,45)$, and that CHRDL1 expression is significantly downregulated in GC tissues and associated with poor survival (39). In the current study, DEGs were confirmed to be enriched in 'signal transduction', 'cell proliferation', and 'negative regulation of cell proliferation' via GO analysis, and enriched in 'Hallmark_Mtorc1_Signaling', 'Hallmark_Kras_Signaling_Up', 'Hallmark_Hedgehog Signaling', and 'Hallmark_Kras_Signaling_Dn' via GSEA analysis. As CLDN11 and CHRDL1 are established factors involved in GC $(31,39)$, it is reasonable to hypothesize that the risk assessment system constructed in the present study is reliable and may prove useful in clarifying the pathogenic process of GC.

However, there are several limitations in the present study. The predictive capability of the present model was established by bioinformatics analysis and it has not been validated in direct experiments. Thus, this model may only provide a direction for further investigation of GC patients with CLIP4 promoter methylation. Additionally, the survival time and pathological staging of different individuals in the two datasets were different, and the therapy data was not available in the validation dataset. Some data bias between training and validation cohort may exist and may impact the accuracy of the analysis. Furthermore, certain information was not available from the original dataset, including the type of chemotherapy or radiotherapy, and whether surgery was performed. However, from Table II, the P-value of chemotherapy in univariate Cox analysis and that of targeted molecular therapy in multivariate Cox analysis are both $>0.05$. Therefore, these two factors were not considered as independent risk factors for OS. Whereas, the risk score established by our study can be considered as independent risk factor for prognosis as the produced $\mathrm{P}<0.05$ in univariate and multivariate Cox analyses. Therefore, the scoring system established in the current study may be useful in predicting the prognosis of GC although further studies are required prior to clinical use.

In summary, the current study study provided a credible risk assessment model for the predicting GC prognosis based on comprehensive bioinformatic analysis of three CLIP4 DNA methylation-associated genes $(C L D N 11, A P O D$ and $C H R D L 1$ ) in two independent datasets. The risk value may be useful as an independent prognostic factor. CLDN11, APOD and $C H R D L 1$ expression was significantly associated with CLIP4 DNA methylation and GC diagnosis and thus, may be potential prognostic biomarkers. 


\section{Acknowledgements}

Not applicable.

\section{Funding}

No funding was received.

\section{Availability of data and materials}

The datasets used and/or analyzed during the current study are available from the corresponding author on reasonable request.

\section{Authors' contributions}

$\mathrm{CH}$ performed data analyses and wrote the manuscript. YZ and CL contributed significantly in data analyses. YK conceived and designed the study. All authors read and approved the final manuscript.

\section{Ethics approval and consent to participate}

In the original articles of the datasets, the trials were approved by the local institutional review boards of all participating centres, and informed consent was obtained from all patients.

\section{Patient consent for publication}

Not applicable.

\section{Competing interests}

The authors declare that they have no competing interests.

\section{References}

1. Ferlay J, Soerjomataram I, Dikshit R, Eser S, Mathers C, Rebelo M, Parkin DM, Forman D and Bray F: Cancer incidence and mortality worldwide: Sources, methods and major patterns in GLOBOCAN 2012. Int J Cancer 136: E359-E386, 2015.

2. Chong Y, Mia-Jan K, Ryu H, Abdul-Ghafar J, Munkhdelger J, Lkhagvadorj S, Jung SY, Lee M, Ji SY, Choi E, et al: DNA methylation status of a distinctively different subset of genes is associated with each histologic Lauren classification subtype in early gastric carcinogenesis. Oncol Rep 31: 2535-2544, 2014.

3. Li Z, Lei H, Luo M, Wang Y, Dong L, Ma Y, Liu C, Song W, Wang F, Zhang J, et al: DNA methylation downregulated mir-10b acts as a tumor suppressor in gastric cancer. Gastric Cancer 18: 43-54, 2015.

4. Ng EK, Chong WW, Jin H, Lam EK, Shin VY, Yu J, Poon TC, Ng SS and Sung JJ: Differential expression of microRNAs in plasma of patients with colorectal cancer: A potential marker for colorectal cancer screening. Gut 58: 1375-1381, 2009.

5. Oue N, Mitani Y, Motoshita J, Matsumura S, Yoshida K, Kuniyasu H, Nakayama H and Yasui W: Accumulation of DNA methylation is associated with tumor stage in gastric cancer. Cancer 106: 1250-1259, 2006.

6. Yamamoto E, Suzuki H, Takamaru H, Yamamoto H, Toyota M and Shinomura Y: Role of DNA methylation in the development of diffuse-type gastric cancer. Digestion 83: 241-249, 2011.

7. Choi J, Cho MY, Jung SY, Jan KM and Kim HS: CpG Island methylation according to the histologic patterns of early gastric adenocarcinoma. Korean J Pathol 2011: 469-476, 2011.

8. Sapari NS, Loh M, Vaithilingam A and Soong R: Clinical potential of DNA methylation in gastric cancer: A meta-analysis. PLoS One 7: e36275, 2012.
9. Pirini F, Noazin S, Jahuira-Arias MH, Rodriguez-Torres S, Friess L, Michailidi C, Cok J, Combe J, Vargas G, Prado W, et al: Early detection of gastric cancer using global, genome-wide and IRF4, ELMO1, CLIP4 and MSC DNA methylation in endoscopic biopsies. Oncotarget 8: 38501-38516, 2017.

10. FeshchenkoEA,SmirnovaEV,SwaminathanG,Teckchandani AM, Agrawal R, Band H, Zhang X, Annan RS, Carr SA and Tsygankov AY: TULA: An SH3- and UBA-containing protein that binds to $\mathrm{c}-\mathrm{Cbl}$ and ubiquitin. Oncogene 23: 4690-4706, 2004.

11. Kowanetz K, Crosetto N, Haglund K, Schmidt MH, Heldin CH and Dikic I: Suppressors of T-cell receptor signaling Sts-1 and Sts-2 bind to Cbl and inhibit endocytosis of receptor tyrosine kinases. J Biol Chem 279: 32786-32795, 2004.

12. Tsygankov AY: TULA-family proteins: A new class of cellular regulators. J Cell Physiol 228: 43-49, 2013.

13. Agrawal R, Carpino N and Tsygankov A: TULA proteins regulate activity of the protein tyrosine kinase Syk. J Cell Biochem 104: 953-964, 2008

14. Chuang JY, Huang YL, Yen WL, Chiang IP, Tsai MH and Tang $\mathrm{CH}$ : Syk/JNK/AP-1 signaling pathway mediates interleukin-6-promoted cell migration in oral squamous cell carcinoma. Int J Mol Sci 15: 545-559, 2014.

15. Luangdilok S, Box C, Patterson L, Court W, Harrington K, Pitkin L, Rhŷs-Evans P, O-charoenrat P and Eccles S: Syk tyrosine kinase is linked to cell motility and progression in squamous cell carcinomas of the head and neck. Cancer Res 67: 7907-7916, 2007.

16. Holz C, Niehr F, Boyko M, Hristozova T, Distel L, Budach V and Tinhofer I: Epithelial-mesenchymal-transition induced by EGFR activation interferes with cell migration and response to irradiation and cetuximab in head and neck cancer cells. Radiother Oncol 101: 158-164, 2011.

17. Lee ST, Feng M, Wei Y, Li Z, Qiao Y, Guan P, Jiang X, Wong $\mathrm{CH}$, Huynh $\mathrm{K}$, Wang $\mathrm{J}$, et al: Protein tyrosine phosphatase UBASH3B is overexpressed in triple-negative breast cancer and promotes invasion and metastasis. Proc Natl Acad Sci USA 110: 11121-11126, 2013.

18. Zouridis H, Deng N, Ivanova T, Zhu Y, Wong B, Huang D, Wu YH, Wu Y, Tan IB, Liem N, et al: Methylation subtypes and large-scale epigenetic alterations in gastric cancer. Sci Transl Med 4: 156ra140, 2012.

19. Ritchie ME, Phipson B, Wu D, Hu Y, Law CW, Shi W and Smyth GK: limma powers differential expression analyses for RNA-sequencing and microarray studies. Nucleic Acids Res 43: e47, 2015

20. Musci RJ, Masyn KE, Uhl G, Maher B, Kellam SG and Ialongo NS: Polygenic score $\mathrm{x}$ intervention moderation: An application of discrete-time survival analysis to modeling the timing of first tobacco use among urban youth. Dev Psychopathol 27: $111-122,2015$.

21. Therneau TM and Grambsch PM: Modeling Survival Data: Extending the Cox Model. Springer, New York, NY, 2000.

22. Bao ZS, Li MY, Wang JY, Zhang CB, Wang HJ, Yan W, Liu YW, Zhang W, Chen L and Jiang T: Prognostic value of a nine-gene signature in glioma patients based on mRNA expression profiling. CNS Neurosci Ther 20: 112-118, 2014.

23. Cheng W, Ren X, Cai J, Zhang C, Li M, Wang K, Liu Y, Han S and Wu A: A five-miRNA signature with prognostic and predictive value for MGMT promoter-methylated glioblastoma patients. Oncotarget 6: 29285-29295, 2015.

24. Zhang CB, Zhu P, Yang P, Cai JQ, Wang ZL, Li QB, Bao ZS, Zhang $\mathrm{W}$ and Jiang T: Identification of high risk anaplastic gliomas by a diagnostic and prognostic signature derived from mRNA expression profiling. Oncotarget 6: 36643-36651, 2015.

25. Huang W, Sherman BT and Lempicki RA: Bioinformatics enrichment tools: Paths toward the comprehensive functional analysis of large gene lists. Nucleic Acids Res 37: 1-13, 2009.

26. Huang W, Sherman BT and Lempicki RA: Systematic and integrative analysis of large gene lists using DAVID bioinformatics resources. Nat Protoc 4: 44-57, 2009.

27. Mootha VK, Lindgren CM, Eriksson K-F, Subramanian A, Sihag S, Lehar J, Puigserver P, Carlsson E, Ridderstråle M, Laurila E, et al: PGC-1alpha-responsive genes involved in oxidative phosphorylation are coordinately downregulated in human diabetes. Nat Genet 34: 267-273, 2003.

28. Subramanian A, Tamayo P, Mootha VK, Mukherjee S, Ebert BL, Gillette MA, Paulovich A, Pomeroy SL, Golub TR, Lander ES, et al: Gene set enrichment analysis: A knowledge-based approach for interpreting genome-wide expression profiles. Proc Natl Acad Sci USA 102: 15545-15550, 2005. 
29. Ahn J, Han KS, Heo JH, Bang D, Kang YH, Jin HA, Hong SJ, Lee JH and Ham WS: FOXC2 and CLIP4 : A potential biomarker for synchronous metastasis of $\leq 7-\mathrm{cm}$ clear cell renal cell carcinomas. Oncotarget 7: 51423-51434, 2016.

30. Hudson TJ, Anderson W, Artez A, Barker AD, Bell C, Bernabé RR, Bhan MK, Calvo F, Eerola I, Gerhard DS, et al; International Cancer Genome Consortium: International network of cancer genome projects. Nature 464: 993-998, 2010.

31. Yang Y,Han L, Yuan Y,Li J,Hei N and Liang H: Gene co-expression network analysis reveals common system-level properties of prognostic genes across cancer types. Nat Commun 5: 3231, 2014.

32. Barrett T, Troup DB, Wilhite SE, Ledoux P, Rudnev D, Evangelista C, Kim IF, Soboleva A, Tomashevsky M, Marshall KA, et al: NCBI GEO: Archive for high-throughput functional genomic data. Nucleic Acids Res 37: D885-D890, 2009.

33. Agarwal R, Mori Y, Cheng Y, Jin Z, Olaru AV, Hamilton JP, David S, Selaru FM, Yang J, Abraham JM, et al: Silencing of claudin-11 is associated with increased invasiveness of gastric cancer cells. PLoS One 4: e8002, 2009.

34. Weinstein RS, Merk FB and Alroy J: The structure and function of intercellular junctions in cancer. Adv Cancer Res 23: 23-89, 1976.

35. Hewitt KJ, Agarwal R and Morin PJ: The claudin gene family: Expression in normal and neoplastic tissues. BMC Cancer 6: 186, 2006.

36. Morin PJ: Claudin proteins in human cancer: Promising new targets for diagnosis and therapy. Cancer Res 65: 9603-9606, 2005.

37. Provost PR, Villeneuve L, Weech PK, Milne RW, Marcel YL and Rassart E: Localization of the major sites of rabbit apolipoprotein D gene transcription by in situ hybridization. J Lipid Res 32: 1959-1970, 1991.

38. Rassart E, Bedirian A, Do Carmo S, Guinard O, Sirois J, Terrisse L and Milne R: Apolipoprotein d. Biochimica et Biophysica Acta (BBA). Protein Struct Mol Enzymol 1482: 185-198, 2000.
39. Muffat J, Walker DW and Benzer S: Human ApoD, an apolipoprotein up-regulated in neurodegenerative diseases, extends lifespan and increases stress resistance in Drosophila. Proc Natl Acad Sci USA 105: 7088-7093, 2008.

40. Terrisse L, Poirier J, Bertrand P, Merched A, Visvikis S, Siest G, Milne R and Rassart E: Increased levels of apolipoprotein D in cerebrospinal fluid and hippocampus of Alzheimer's patients. J Neurochem 71: 1643-1650, 1998

41. Pei YF, Zhang YJ, Lei Y, Wu DW, Ma TH and Liu XQ: Hypermethylation of the CHRDL1 promoter induces proliferation and metastasis by activating Akt and Erk in gastric cancer. Oncotarget 8: 23155-23166, 2017.

42. Wang Z, Shen Z, Li Z, Duan J, Fu S, Liu Z, Bai H, Zhang Z, Zhao J, Wang X, et al: Activation of the BMP-BMPR pathway conferred resistance to EGFR-TKIs in lung squamous cell carcinoma patients with EGFR mutations. Proc Natl Acad Sci USA 112: 9990-9995, 2015.

43. Lei H, Wang J, Lu P, Si X, Han K, Ruan T and Lu J: BMP10 inhibited the growth and migration of gastric cancer cells. Tumour Biol 37: 3025-3031, 2016.

44. Sawala A, Sutcliffe C and Ashe HL: Multistep molecular mechanism for bone morphogenetic protein extracellular transport in the Drosophila embryo. Proc Natl Acad Sci USA 109: $11222-11227,2012$

45. Watanabe T, Nagai A, Sheikh AM, Mitaki S, Wakabayashi K, Kim SU, Kobayashi S and Yamaguchi S: A human neural stem cell line provides neuroprotection and improves neurological performance by early intervention of neuroinflammatory system. Brain Res 1631: 194-203, 2016.

(i) $\mathrm{C}$ This work is licensed under a Creative Commons Attribution-NonCommercial-NoDerivatives 4.0 International (CC BY-NC-ND 4.0) License. 